\title{
Influence of foliar application of polyamines on growth, gas-exchange characteristics, and chlorophyll fluorescence in Bakraii citrus under saline conditions
}

\author{
D. KHOSHBAKHT ${ }^{*}+$, M.R. ASGHARI ${ }^{*}$, and M. HAGHIGHI ${ }^{* *}$ \\ Department of Horticultural Science, College of Agriculture, University of Urmia, West Azarbaijan, Iran* \\ Department of Horticulture Science, College of Agriculture, Isfahan University of Technology, Isfahan, Iran**
}

\begin{abstract}
The effects of polyamines (PAs) on salt stress in Bakraii (Citrus reticulata $\times$ Citrus limetta) seedlings were studied. Foliar treatments by putrescine (Put), spermidine (Spd), and spermine (Spm) $(0,0.5$, and $1 \mathrm{mM})$ were applied during the salinity period $(0$ and $75 \mathrm{mM}$ of $\mathrm{NaCl})$. PA-treated seedlings showed a lower content of $\mathrm{Na}^{+}$and $\mathrm{Cl}^{-}$in leaves. Application of PAs increased net photosynthetic rate in salt-stressed plants and it contributed to the enhanced growth parameters. PAs application considerably induced growth improvement in Bakraii seedlings which was found to be associated with reduced electrolyte leakage, increased relative water content, chlorophyll fluorescence parameters, activities of key antioxidant enzymes, as well as increased photosynthetic pigment concentration under saline regime. These results showed the promising use of PAs, especially of Spd and Spm, for reducing the negative effects of salinity stress and improving the growth of citrus seedlings.
\end{abstract}

Additional key words: abiotic stress; biomass; citrus; oxidative stress; photosystem II efficiency.

\section{Introduction}

Salinity is among the most significant, environmental factors responsible for substantial losses in agricultural production worldwide. It is also one of the most serious problems confronting sustainable agriculture in irrigated production systems in arid and semiarid regions (Ravindran et al. 2007). Nearly $20 \%$ of the world cultivated area and about half of the world irrigated lands are affected by this stress (Munns and Tester 2008). The progressive salinization of agricultural land is considered as the major environmental factor limiting plant growth and productivity of the arid and semiarid areas (Sudhir and Murthy 2004).

Citrus is grown commercially in over 50 countries and ranks as the top fruit crop in world production (GarcíaSánchez et al. 2002). Citrus plants are known to be sensitive to salts (Anjum 2007) because of the specific toxicity of $\mathrm{Cl}^{-}$and/or $\mathrm{Na}^{+}$and to the osmotic effect caused by the high concentration of salts (García-Sánchez 2000).

Salinity causes various injuries in plants, such as tissue burning, yield reduction, and finally plant death (RomeroAranda et al. 2001), causes leaf senescence, reduction of PSII activities (Nishihara et al. 2003), enhancing membrane permeability (Dhindsa et al. 1981), nutritional imbalance, and toxicity (Grattan and Grieve 1992). $\mathrm{NaCl}$ inhibits net photosynthetic rate $\left(P_{\mathrm{N}}\right)$ as a consequence of osmotic stress, which leads to a decrease in water potential and stomatal conductance $\left(g_{\mathrm{s}}\right)$, sugar accumulation, which causes feedback inhibition, and ion toxicity (e.g., an excess of $\mathrm{Na}^{+}$and $\mathrm{Cl}^{-}$) accompanied by a reduction of $\mathrm{K}^{+}$and $\mathrm{Ca}^{2+}$ (Koshbakht and Asgharei 2015). The reduction in $P_{\mathrm{N}}$ caused by salt stress depends on the kind and concentration of the salt, how the stress occurs, and the sensitivity of a plant (Lu et al. 2003). Salinity also induces generation of reactive oxygen species (ROS), such as superoxide,

Received 12 October 2016, accepted 13 February 2017, published as online-first 6 April 2017.

${ }^{+}$Corresponding author; tel: +98 3113913447 , fax: +98 311 3913356, e-mail: davod.khoshbakht@gmail.com

Abbreviations: $C_{\mathrm{a}}$ - atmospheric $\mathrm{CO}_{2}$ concentration; $\mathrm{CAT}$ - catalase; $\mathrm{Chl}$ - chlorophyll; $C_{\mathrm{i}}$ - intercellular $\mathrm{CO}_{2}$ concentration; $\mathrm{DM}$ - dry mass; EL - electrolyte leakage; FM - fresh mass; $\mathrm{F}_{0}$ - minimal fluorescence yield of the dark-adapted state; $\mathrm{F}_{\mathrm{m}}$ - maximal fluorescence yield of the dark-adapted state; $F_{v}$ - variable fluorescence; $F_{v} / F_{m}-$ maximum photochemical efficiency of PSII; $g_{s}-$ stomatal conductance; TLA - total leaf area; LN - number of leaves per plant; NPQ - nonphotochemical quenching; PAs - polyamine; Put putrescine; $P_{\mathrm{N}}$ - net photosynthetic rate; $\mathrm{qP}$ - photochemical quenching; RWC - relative water content; ROS - reactive oxygen species; SL - length of shoot; SOD - superoxide dismutase; Spd - spermidine; Spm - spermine; S - salinity stress; TDM - total plant dry mass; Acknowledgements: Here we would like to thank to the Department of Horticulture, College of Agriculture, University of Urmia for financial support of the research. 
hydrogen peroxide, singlet oxygen, and hydroxyl radicals in plants (Leshem et al. 2007). ROS can cause toxic reactions, such as lipid peroxidation, protein degradation, and DNA mutation (McCord 2000). Salt stress alters the critical balance between the production of ROS and the quenching activity of antioxidants, resulting in oxidative stress that causes damage to plants (Hernández et al. 1999). Antioxidant enzymes, namely superoxide dismutase (SOD), catalase (CAT), ascorbate peroxidase, and glutathione peroxidase, and nonenzymatic antioxidants, such as ascorbic acid, glutathione, $\alpha$-tocopherol, carotenoids, phenolics, and proline, which play a key role in quenching ROS, are implicated in stress tolerance (Hernández et al. 1999).

PAs, such as Put, Spd, and Spm, are plant growth regulators and low-molecular-mass polycations occurring in all living organisms (Kusano et al. 2008). Some reports have indicated the relationships between PAs and environmental stress (Galston et al. 1997, Bouchereau et al. 1999). Biosynthesis of PAs may be an integral part of

\section{Materials and methods}

Plant material, growth conditions, salinity, and PAs treatments: The experiment was conducted under greenhouse conditions. Seeds of Iranian mandarin Bakraii (Citrus reticulata $\times$ Citrus limetta) were sown in air-dried soil (15 kg in each pot). The texture of the soil was loamy clay ( $\mathrm{pH} 7.1$, EC of $\left.0.54 \mathrm{dS} \mathrm{m}^{-1}\right)$. After germination, seedlings (six-month-old plants) were transplanted into $30-\mathrm{cm}$ wide plastic pots containing fine sand, then placed in a greenhouse at $25-28 / 17^{\circ} \mathrm{C}$ (day/night) temperature, maximum PAR of $800 \mu \mathrm{mol}$ (photon) $\mathrm{m}^{-2} \mathrm{~s}^{-1}, 60 / 70 \pm 5 \%$ relative humidity, and a $16-\mathrm{h}$ photoperiod. Plants were irrigated at 2-d intervals with $0.5 \mathrm{~L}$ of water and fertilized with a commercial water soluble fertilizer containing macro- and micronutrients (Floral Mixed fertilizer, IFO, Italy). After the acclimation period of at least six months, (twelve months after seed germination), seedlings were sprayed at two-week interval (for the first time it was two weeks before the onset of salinity treatments) with 0 (control), 0.50, or $1.00 \mathrm{mM}$ Put, Spd, and Spm, respectively, until both sides of all the leaves were completely wet. Tween-20 at $1 \%(\mathrm{v} / \mathrm{v})$ was added to PAs solutions and control treatment as a surfactant. Two weeks after the foliar application of PAs, all seedlings were exposed to salt treatments. For salt treatments, $75 \mathrm{mM} \mathrm{NaCl}$ was added to the pots at 3 -d intervals using $0.5 \mathrm{~L}$ of irrigation water. To avoid osmotic shock, the $\mathrm{NaCl}$ concentration was increased gradually. After $60 \mathrm{~d}$ of the salt treatment, various analyses were performed.

Growth characteristics: At the end of experiment, after measuring the length of shoot (SL), plants were harvested and number of leaves per plant (LN) was counted. Then plants were washed with distilled water to remove adhering foreign particles. The shoots and roots fresh mass plant's response to salinity stress (Alcazar et al. 2010). Increasing polyamine biosynthesis might protect the plants from salinity by removing free radicals, maintaining membrane and cellular structures, keeping a cation-anion balance (Bouchereau et al. 1999), regulation of ion channels, and induction of ATP synthesis (Lopatin et al. 1994). Both mono- and dicotyledonous plants increase the accumulation of endogenous PAs under salt stress, and the pattern of PA metabolism in response to salinity seems to be dependent on plant defense systems and/or duration of exposure to salt stress. Exogenous PA application has been proposed as a convenient and effective approach for combating the salt tolerance of plants and ultimately improving crop productivity under high salinity (Chattopadhayay et al. 2003).

Therefore, the objectives of this work were to determine (1) the effect of salt stress and polyamines on the morphological and physiological changes in citrus plants, and (2) whether a foliar supply of PAs to Bakraii seedlings might be a strategy for increasing their salt tolerance.

(FM) were recorded, and the samples were dried separately at $80^{\circ} \mathrm{C}$ for $48 \mathrm{~h}$ and their dry masses (DM) were then recorded.

Leaf tissue mineral analysis: At the end of experiment, leaves of each plant were separated and washed with deionized water. Tissues were oven-dried at $70^{\circ} \mathrm{C}$ for $3 \mathrm{~d}$ and dried parts were milled to a powder for mineral nutrient analysis. Ground samples were ashed in porcelain crucibles at $550^{\circ} \mathrm{C}$ for $6 \mathrm{~h}$. The white ash was treated in $2 \mathrm{ml}$ of hot $\mathrm{HCl}$, filtered into a $100-\mathrm{ml}$ volumetric flask, and made up to $100 \mathrm{ml}$ with distilled water. Concentration of $\mathrm{Na}^{+}$and $\mathrm{K}^{+}$were determined by flame photometry (Model PFP7, Jenway, UK). $\mathrm{Ca}^{2+}$ and $\mathrm{Mg}^{2+}$ concentrations were measured by using an atomic absorption spectrophotometer (Perkin Elmper Analyst Model 200, USA). $\mathrm{Cl}^{-}$ was extracted from $500 \mathrm{mg} \mathrm{DM}$ of leaf tissue with $0.1 \mathrm{~mol}\left(\mathrm{HNO}_{3}\right) \mathrm{L}^{-1}, 0.1 \mathrm{M} \mathrm{HNO}_{3}$ in $10 \%(\mathrm{v} / \mathrm{v})$ glacial acetic acid, and samples were incubated overnight at room temperature and then filtered. Finally, $\mathrm{Cl}^{-}$concentration was determined by silver ion titration (Moya et al. 1999).

Assay of SOD and CAT: Frozen leaf samples (1 g) were homogenized in $50 \mathrm{mM}$ sodium phosphate buffer $(\mathrm{pH} 7.8$ for SOD and $\mathrm{pH} 7$ for CAT). Then samples were centrifuged at $12,000 \times \mathrm{g}$ for $20 \mathrm{~min}$ at $4^{\circ} \mathrm{C}$. The supernatant was used to measure the activity of the enzymes. SOD (EC 1.15.1.1) activity was measured according to the method described by Giannopolitis and Ries (1977). One unit of SOD activity was defined as the amount of enzyme required to cause $50 \%$ inhibition of the rate of $p$-nitroblue tetrazolium chloride reduction at $560 \mathrm{~nm}(U-2000$, Hitachi Instruments, Tokyo, Japan). SOD activity was expressed as unit $\mathrm{min}^{-1} \mathrm{mg}^{-1}$ (protein). CAT (EC 1.11.1.6) activity 
was determined according to Cakmak and Marschner (1992). The reaction mixture in a total volume of $2 \mathrm{ml}$ contained sodium phosphate buffer $(25 \mathrm{mM}, \mathrm{pH} 7)$ and $\mathrm{H}_{2} \mathrm{O}_{2}(10 \mathrm{mM})$. The reaction was initiated by the addition of $100 \mu \mathrm{l}$ of enzyme extract and the activity was measured by determining the initial rate of disappearance of $\mathrm{H}_{2} \mathrm{O}_{2}$ at $240 \mathrm{~nm}$ using the extinction coefficient of $39.4 \mathrm{mM}^{-1} \mathrm{~cm}^{-1}$ with a spectrophotometer $(U-2000$, Hitachi Instruments, Tokyo, Japan) for $30 \mathrm{~s}$. One unit of CAT activity was defined as $1 \mu \mathrm{mol} \mathrm{H}_{2} \mathrm{O}_{2}$ (decomposed) $\mathrm{min}^{-1} \mathrm{mg}^{-1}$ (protein).

Starch analysis: Fresh leaf sample (the youngest fullyexpanded leaves) was homogenized in $80 \%$ ethanol. After extraction, the concentration of starch was measured by the method of Hedge and Hofreiter (1962). The absorbance was measured at $630 \mathrm{~nm}$ using a spectrophotometer (U-2000, Hitachi Instruments, Tokyo, Japan). Glucose was used as standard solution.

Assay of protein: The protein was measured using bovine serum as standard according to the method of Bradford (1976).

Electrolyte leakage (EL) was used to assess membrane permeability. EL was measured using a conductivity meter (CC-501; Elmetron, Zabrze, Poland). Six leaf discs were taken from the youngest fully-expanded leaf on one randomly chosen plant per replicate sample (pot). After three washes with distilled water to remove surface contamination, six leaf discs were then placed in test-tube containing $10 \mathrm{ml}$ of distilled water. These samples were incubated for $24 \mathrm{~h}$ on a shaker at room temperature. The electrical conductivity (EC) of the solution (EC1) was read after incubation. The same samples were then placed in an autoclave at $120^{\circ} \mathrm{C}$ for $20 \mathrm{~min}$ and the second $\mathrm{EC}$ reading (EC2) was taken after cooling the solution to room temperature. EL was then calculated as EC1/EC2, and expressed as a percentage (Lutts et al. 1995).

Relative water content (RWC) of leaves was determined following the method suggested by Barrs and Weatherley (1962). Ten leaf discs of $1 \mathrm{~cm}$ in diameter were cut from the youngest fully-expanded leaf, using a leaf punch. Leaf discs were weighed (FM) and washed three times with double distilled water and placed into a $10-\mathrm{ml}$ conical flask. Leaf discs were immersed in $10 \mathrm{ml}$ distilled water for $4 \mathrm{~h}$ at $4^{\circ} \mathrm{C}$ in dark. Turgid mass (TM) of leaf discs was then measured and samples were dried in hot air oven at $70^{\circ} \mathrm{C}$ until constant mass (DM) was achieved. RWC was estimated using the following equation:

$$
\operatorname{RWC}(\%)=[(F M-D M) /(T M-D M)] \times 100 .
$$

Chlorophyll (Chl) content was determined according to Lichtenthaler (1987). After gas-exchange measurements, Chl was extracted from $500 \mathrm{mg}$ of leaf, which was chosen from the same leaf used for $P_{\mathrm{N}}$ measurements (avoiding major veins) using $80 \%$ aqueous acetone. Absorbance of centrifuged extracts was measured at 645 and $663 \mathrm{~nm}$ using a spectrophotometer (U-2000, Hitachi Instruments, Tokyo, Japan).

Proline (Pro) was determined according to the method described by Bates et al. (1973). Seedlings (0.5 g fresh shoot material) were homogenized in $10 \mathrm{ml}$ of $3 \%(\mathrm{v} / \mathrm{v})$ aqueous sulphosalicylic acid and filtered through a Whatman No. 2 filter paper. The filtrate $(2 \mathrm{ml})$ was then mixed with $2 \mathrm{ml}$ of acid-ninhydrin reagent and $2 \mathrm{ml}$ of glacial acetic acid in a test-tube and the mixture was placed in a water bath for $1 \mathrm{~h}$ at $100^{\circ} \mathrm{C}$. The reaction mixture was extracted with $4 \mathrm{ml}$ of toluene, and the chromophorecontaining toluene fraction was aspirated, cooled to room temperature, and its absorbance measured at $520 \mathrm{~nm}$ using a spectrophotometer (U-2000, Hitachi Instruments, Tokyo, Japan). Appropriate Pro standards (Sigma Chemical Co., St. Louis, MO, USA) were included in order to calculate the concentration of Pro in each shoot tissue sample.

Chl fluorescence was measured in the dark- and lightadapted leaves between 9:00-11:00 h, with a portable fluorometer (PAM-2500, Walz, Effeltrich, Germany). After 30 min of dark adaptation, $F_{\mathrm{v}} / \mathrm{F}_{\mathrm{m}}$ was calculated as $\left(F_{m}-F_{0}\right) / F_{m}$, where $F_{m}$ [induced by a short pulse $(0.6 \mathrm{~s})$ of saturating light of $3,450 \mu \mathrm{mol}$ (photon) $\mathrm{m}^{-2} \mathrm{~s}^{-1}$ ] and $\mathrm{F}_{0}$ were the maximal and minimal fluorescence, respectively (Genty et al. 1989). After 4 min of illumination with continuous red, nonsaturating actinic light $[447 \mu \mathrm{mol}$ (photon) $\left.\mathrm{m}^{-2} \mathrm{~s}^{-1}\right]$ and saturating pulses every $25 \mathrm{~s}$, the maximum $\left(\mathrm{F}_{\mathrm{m}}{ }^{\prime}\right)$ and the steady state $\left(\mathrm{F}_{\mathrm{s}}\right)$ fluorescence signals were measured in the light-adapted leaves. Then, the actinic light was turned off and the far-red pulse was applied to obtain the minimal fluorescence after the PSI excitation $\left(F_{0}{ }^{\prime}\right)$. $q_{p}$ was calculated as $\left(F_{m}{ }^{\prime}-F_{s}\right) /\left(F_{m}{ }^{\prime}-F_{0}{ }^{\prime}\right)($ van Kooten and Snel 1990). NPQ, which is proportional to the rate constant of the thermal energy dissipation, was estimated as $\left(\mathrm{F}_{\mathrm{m}}-\mathrm{F}_{\mathrm{m}}{ }^{\prime}\right) / \mathrm{F}_{\mathrm{m}}{ }^{\prime}$ (Bilger et al. 2001).

Gas-exchange parameters [net photosynthetic rate $\left(P_{\mathrm{N}}\right)$, intercellular $\mathrm{CO}_{2}$ concentration $\left(C_{\mathrm{i}}\right)$, and stomatal conductance $\left(g_{\mathrm{s}}\right)$ ] were measured between 9:00-11:00 $\mathrm{h}$ at the end of the experiment using a portable photosynthetic system (LI-6200, LI-COR Inc., Lincoln, NE, USA). Top fully expanded leaf was clamped to the leaf chamber and the observations were recorded when relative humidity $(\mathrm{RH})$ and atmospheric $\mathrm{CO}_{2}$ concentration $\left(C_{\mathrm{a}}\right)$ reached a stable value. $\mathrm{PAR}$, air temperature, $\mathrm{RH}$, and $\mathrm{CO}_{2}$ concentration inside the sensor head were set at 1,300 $\pm 100 \mu \mathrm{mol}$ (photon) $\mathrm{m}^{-2} \mathrm{~s}^{-1}, 31 \pm 2^{\circ} \mathrm{C}, 60 \%$, and $335-340 \mu \mathrm{mol} \mathrm{m}{ }^{-1}$, respectively, when measuring $P_{\mathrm{N}}$.

Statistical analysis: All data are presented as means \pm SD of five replicate seedlings. Statistical analyses were performed by one-way analysis of variance (ANOVA) using the $S A S$ program version 9.1 (SAS Inc., Cary, NC, USA). Differences between treatments were separated by the least significant differences $(L S D)$ test at $P<0.05$. 


\section{Results}

Growth parameters: SL of salt-treated Bakraii seedlings decreased with an increasing salt content, but different foliar PAs improved the growth of the PAs-treated plants. The maximum increase of SL was recorded for the plants treated by $\mathrm{Spd}(0.5 \mathrm{mM})$ while the minimum SL was recorded for the seedlings at $75 \mathrm{mM} \mathrm{NaCl}$ without PAs treatments (Table 1). Spd was the most effective PAs in improving the SL in salinity treatment. TPFM and TPDM of the seedlings were affected by salinity. Increase of salinity from 0 to $75 \mathrm{mM}$ significantly decreased the TPFM and TPDM. PAs treatments, especially Spm and Spd, caused a significant increase in TPFM and TPDM in comparison to Put treatment. The highest TPDM was recorded for the Spm- and Spd-treated seedlings with $75 \mathrm{mM} \mathrm{NaCl}$. Although Spm increased the TPDM at $75 \mathrm{mM} \mathrm{NaCl}$, there was no significant difference between the application of Spd and Spm at $0.5 \mathrm{mM}$. The lowest TPFM and TPDM were observed for the seedlings under $75 \mathrm{mM} \mathrm{NaCl}$ without PAs treatments (Table 1). The LN was significantly affected by increasing the salt stress from 0 to $75 \mathrm{Mm}$, with $\mathrm{NaCl}$ stress significantly decreasing the LN (Table 1). The leaves of seedlings treated with salt showed chlorosis and toxicity symptoms leading to leaf abscission. Application of PAs, especially of Spd $(0.5 \mathrm{mM})$, significantly prevented the leaf abscission. The maximum LN was related to Spd $(0.5 \mathrm{mM})$ under $75 \mathrm{mM}$ $\mathrm{NaCl}$, while the minimum was recorded for nontreated seedlings at $75 \mathrm{mM}$ salt stress (Table 1).
Physiological and biochemical parameters: Leaf $\mathrm{Na}^{+}$ and $\mathrm{Cl}^{-}$concentrations were significantly elevated with $\mathrm{NaCl}$ (Table 2). Salinity increased $\mathrm{K}^{+}$and decreased $\mathrm{Ca}^{2+}$ and $\mathrm{Mg}^{2+}$ concentrations in leaves. The lowest $\mathrm{Ca}^{2+}$ and $\mathrm{Mg}^{2+}$ contents were found in control plants, not treated by PAs, at salt conditions. Leaf $\mathrm{Ca}^{2+}, \mathrm{Mg}^{2+}$, and $\mathrm{K}^{+}$were significantly elevated by the exogenous application of PAs. PAs resulted in the significant reduction of the $\mathrm{Na}^{+}$ and $\mathrm{Cl}^{-}$contents of the leaves. Spd significantly decreased the $\mathrm{Na}^{+}$and $\mathrm{Cl}^{-}$contents in leaves under $75 \mathrm{mM} \mathrm{NaCl}$ (Table 2). Salt stress significantly increased EL, with maximum values observed in plants grown with $75 \mathrm{mM}$ $\mathrm{NaCl}$. The application of PAs significantly decreased EL in leaf discs, with the largest decrease in EL measured when $0.50 \mathrm{mM}$ Spd was applied (Table 3). RWC and Chl were significantly affected by both salinity and PAs pretreatment (Table 3). Increasing the concentrations of $\mathrm{NaCl}$ from 0 to $75 \mathrm{mM}$ lowered RWC and $\mathrm{Chl}$ in Bakraii plants (Table 3). All concentrations of PAs increased RWC and Chl significantly compared with the control. However, $0.50 \mathrm{mM}$ Spd was the most effective PA (Table 3). Increasing the concentration of salt resulted in higher leaf Pro (Table 3). The highest Pro was recorded for PAnontreated seedlings at $75 \mathrm{mM} \mathrm{NaCl}$, while the lowest content was related to the Spd-treated seedlings $(0.5 \mathrm{mM})$ under $75 \mathrm{mM} \mathrm{NaCl}$. In $75 \mathrm{mM} \mathrm{NaCl}$, the amount of Pro in Bakraii leaves decreased by application of PAs and Spd showed the highest impact (Table 3). The protein and

Table 1. Growth characteristics of Iranian mandarin Bakraii seedlings treated with PAs and NaCl. Each value was mean \pm SD $(n=5)$. Mean values followed by the same letters within a column are not significantly different at $p<0.05$. Analysis of variance $($ ANOVA $) ; * * *$ - significant $(P<0.001) ; * *$ - significant $(P<0.01)$. C - control; LN - leaf number; PAs - polyamine; Put - putrescine; $\mathrm{S}$ - $75 \mathrm{mM} \mathrm{NaCl}$; SL - shoot length; Spd - spermidine; Spm - spermine; TPDM - total plant dry mass; TPFDM - total plant fresh mass; CV - coefficient of variation.

\begin{tabular}{llcll}
\hline Treatment & LN & TPDM $[\mathrm{g}]$ & TPFM $[\mathrm{g}]$ & SL $[\mathrm{cm}]$ \\
\hline C & $30.00 \pm 2.44^{\mathrm{bcd}}$ & $14.91 \pm 1.63^{\mathrm{c}}$ & $49.71 \pm 2.44^{\mathrm{c}}$ & $37.41 \pm 1.70^{\mathrm{cd}}$ \\
Put 0.5 & $30.10 \pm 1.63^{\mathrm{bcd}}$ & $15.80 \pm 2.44^{\mathrm{bc}}$ & $51.65 \pm 0.82^{\mathrm{bc}}$ & $38.97 \pm 1.70^{\mathrm{bc}}$ \\
Put 1 & $27.70 \pm 1.63^{\mathrm{de}}$ & $15.31 \pm 1.63^{\mathrm{bc}}$ & $50.39 \pm 1.63^{\mathrm{bc}}$ & $38.06 \pm 1.50^{\mathrm{bcd}}$ \\
Spd 0.5 & $37.62 \pm 0.82^{\mathrm{a}}$ & $18.40 \pm 1.63^{\mathrm{a}}$ & $55.81 \pm 4.08^{\mathrm{a}}$ & $41.91 \pm 1.63^{\mathrm{a}}$ \\
Spd 1 & $28.80 \pm 1.63^{\mathrm{cd}}$ & $15.23 \pm 1.61^{\mathrm{bc}}$ & $52.90 \pm 2.45^{\mathrm{abc}}$ & $39.17 \pm 1.60^{\mathrm{bc}}$ \\
Spm 0.5 & $32.60 \pm 1.53^{\mathrm{b}}$ & $16.81 \pm 1.60^{\mathrm{ab}}$ & $53.70 \pm 2.44^{\mathrm{ab}}$ & $39.22 \pm 1.63^{\mathrm{bc}}$ \\
Spm 1 & $30.88 \pm 2.44^{\mathrm{bc}}$ & $15.05 \pm 0.19^{\mathrm{c}}$ & $51.20 \pm 0.82^{\mathrm{bc}}$ & $40.29 \pm 1.70^{\mathrm{ab}}$ \\
S & $16.00 \pm 2.40^{\mathrm{h}}$ & $5.90 \pm 0.82^{\mathrm{g}}$ & $20.08 \pm 1.63^{\mathrm{h}}$ & $26.95 \pm 1.54^{\mathrm{g}}$ \\
S + Put 0.5 & $24.00 \pm .82^{\mathrm{fg}}$ & $10.88 \pm 0.82^{\mathrm{de}}$ & $34.31 \pm 3.26^{\mathrm{e}}$ & $34.76 \pm 1.60^{\mathrm{e}}$ \\
S + Put 1 & $23.60 \pm 2.04^{\mathrm{fg}}$ & $8.65 \pm 0.82^{\mathrm{f}}$ & $25.15 \pm 4.08^{\mathrm{g}}$ & $29.97 \pm 1.82^{\mathrm{f}}$ \\
S + Spd 0.5 & $25.80 \pm 1.63^{\mathrm{ef}}$ & $12.45 \pm 1.63^{\mathrm{d}}$ & $42.89 \pm 2.45^{\mathrm{d}}$ & $35.87 \pm 1.70^{\mathrm{de}}$ \\
S + Spd 1 & $23.16 \pm 2.32^{\mathrm{fg}}$ & $10.09 \pm 0.82^{\mathrm{ef}}$ & $33.97 \pm 2.44^{\mathrm{e}}$ & $33.55 \pm 1.60^{\mathrm{e}}$ \\
S + Spm 0.5 & $23.20 \pm .80^{\mathrm{fg}}$ & $11.54 \pm 0.80^{\mathrm{de}}$ & $40.60 \pm 3.26^{\mathrm{d}}$ & $33.83 \pm 1.29^{\mathrm{e}}$ \\
S + Spm 1 & $22.00 \pm 1.03^{\mathrm{g}}$ & $8.52 \pm 1.63^{\mathrm{f}}$ & $29.05 \pm 0.82^{\mathrm{f}}$ & $30.98 \pm 1.70^{\mathrm{f}}$ \\
ANOVA & & & & \\
Treatment & $* * *$ & $* *$ & $* *$ \\
CV [\%] & 19.4 & 8.8 & 9.7 & 6.8 \\
\hline
\end{tabular}


Table 2. Leaf $\mathrm{Cl}^{-}, \mathrm{Na}^{+}, \mathrm{K}^{+}, \mathrm{Ca}^{2+}, \mathrm{Mg}^{2+}$ contents in Iranian mandarin Bakraii seedlings treated with PAs and $\mathrm{NaCl}$. Each value was mean $\pm \mathrm{SD}(n=5)$. Mean values followed by the same letters within a column are not significantly different at $p<0.05$. Analysis of variance $($ ANOVA $) ; * * *$ - significant $(P<0.001) ; * *$ - significant $(P<0.01)$. C - control; PAs - polyamine; Put - putrescine; $\mathrm{S}-75 \mathrm{mM} \mathrm{NaCl}$; $\mathrm{Spd}$ - spermidine; $\mathrm{Spm}$ - spermine; $\mathrm{CV}$ - coefficient of variation.

\begin{tabular}{llllll}
\hline Treatment & $\mathrm{Cl}^{-}[\%]$ & $\mathrm{Na}^{+}[\%]$ & $\mathrm{K}^{+}[\%]$ & $\mathrm{Ca}^{2+}[\%]$ & $\mathrm{Mg}^{2+}[\%]$ \\
\hline C & $0.73 \pm 0.02^{\mathrm{f}}$ & $0.17 \pm 0.02^{\mathrm{f}}$ & $2.10 \pm 0.16^{\mathrm{e}}$ & $2.50 \pm 0.41^{\mathrm{c}}$ & $0.38 \pm 0.02^{\mathrm{abcd}}$ \\
Put 0.5 & $0.50 \pm 0.08^{\mathrm{g}}$ & $0.13 \pm 0.01^{\mathrm{f}}$ & $2.48 \pm 0.24^{\mathrm{d}}$ & $2.67 \pm 0.16^{\mathrm{bc}}$ & $0.42 \pm 0.16^{\mathrm{abc}}$ \\
Put 1 & $0.59 \pm 0.08^{\mathrm{fg}}$ & $0.14 \pm 0.02^{\mathrm{f}}$ & $2.47 \pm 0.16^{\mathrm{d}}$ & $2.53 \pm 0.16^{\mathrm{c}}$ & $0.38 \pm 0.20^{\mathrm{abcd}}$ \\
Spd 0.5 & $0.50 \pm 0.08^{\mathrm{g}}$ & $0.11 \pm 0.01^{\mathrm{f}}$ & $2.80 \pm 0.16^{\mathrm{bcd}}$ & $3.08 \pm 0.16^{\mathrm{a}}$ & $0.48 \pm 0.08^{\mathrm{a}}$ \\
Spd 1 & $0.52 \pm 0.08^{\mathrm{fg}}$ & $0.12 \pm 0.02^{\mathrm{f}}$ & $2.60 \pm 0.16^{\mathrm{cd}}$ & $2.83 \pm 0.16^{\mathrm{ab}}$ & $0.44 \pm 0.07^{\mathrm{ab}}$ \\
Spm 0.5 & $0.54 \pm 0.08^{\mathrm{fg}}$ & $0.11 \pm 0.02^{\mathrm{f}}$ & $2.70 \pm 0.4^{\mathrm{bcd}}$ & $2.79 \pm .24^{\mathrm{abc}}$ & $0.42 \pm 0.09^{\mathrm{abc}}$ \\
Spm 1 & $0.57 \pm 0.08^{\mathrm{fg}}$ & $0.13 \pm 0.02^{\mathrm{f}}$ & $2.50 \pm 0.4^{\mathrm{d}}$ & $2.63 \pm 0.16^{\mathrm{bc}}$ & $0.45 \pm 0.04^{\mathrm{ab}}$ \\
S & $4.10 \pm 0.08^{\mathrm{a}}$ & $2.10 \pm 0.02^{\mathrm{a}}$ & $2.50 \pm 0.24^{\mathrm{d}}$ & $1.50 \pm 0.24^{\mathrm{f}}$ & $0.20 \pm 0.08^{\mathrm{e}}$ \\
S + Put 0.5 & $2.52 \pm 0.08^{\mathrm{d}}$ & $1.40 \pm 0.32^{\mathrm{bc}}$ & $2.90 \pm 0.24^{\mathrm{bbc}}$ & $1.73 \pm 0.16^{\mathrm{def}}$ & $0.30 \pm 0.16^{\mathrm{cde}}$ \\
S + Put 1 & $3.20 \pm 0.16^{\mathrm{b}}$ & $1.60 \pm 0.16^{\mathrm{b}}$ & $2.70 \pm 0.16^{\mathrm{bcd}}$ & $1.58 \pm 0.24^{\mathrm{ef}}$ & $0.29 \pm 0.02^{\mathrm{de}}$ \\
S + Spd 0.5 & $2.00 \pm 0.32^{\mathrm{e}}$ & $0.90 \pm 0.08^{\mathrm{e}}$ & $3.20 \pm 0.16^{\mathrm{a}}$ & $1.92 \pm 0.16^{\mathrm{d}}$ & $0.34 \pm 0.06^{\mathrm{bcd}}$ \\
S + Spd 1 & $2.90 \pm 0.08^{\mathrm{c}}$ & $1.20 \pm 0.16^{\mathrm{cd}}$ & $3.00 \pm 0.24^{\mathrm{ab}}$ & $1.63 \pm 0.16^{\mathrm{def}}$ & $0.30 \pm 0.08^{\mathrm{cde}}$ \\
S + Spm 0.5 & $2.40 \pm 0.32^{\mathrm{d}}$ & $1.10 \pm 0.08^{\mathrm{de}}$ & $3.00 \pm 0.24^{\mathrm{ab}}$ & $1.86 \pm 0.16^{\mathrm{de}}$ & $0.33 \pm 0.08^{\mathrm{bcd}}$ \\
S + Spm 1 & $3.20 \pm 0.16^{\mathrm{b}}$ & $1.30 \pm 0.24^{\mathrm{cd}}$ & $2.80 \pm 0.16^{\mathrm{bcd}}$ & $1.65 \pm 0.16^{\mathrm{def}}$ & $0.30 \pm 0.02^{\mathrm{cde}}$ \\
ANOVA & & & & $*$ & $*$ \\
Treatment & $* * *$ & $* * *$ & $* *$ & 11.6 & 23.4 \\
CV [\%] & 14.4 & 9.7 & 9.3 & & \\
\hline
\end{tabular}

Table 3. Leaf characteristics of Iranian mandarin Bakraii seedlings treated with PAs and NaCl. Each value was mean $\pm \mathrm{SD}(n=5)$. Mean values followed by the same letters within a column are not significantly different at $p<0.05$. Analysis of variance $(A N O V A)$; *** - significant $(P<0.001) ; * *$ - significant $(P<0.01)$. C - control; Chl - total chlorophyll concentration; EL - electrolyte leakage; PAs polyamine; Pro - proline content; Put - putrescine; S - $75 \mathrm{mM} \mathrm{NaCl}$; RWC - relative water content; Spd - spermidine; Spm - spermine; $\mathrm{CV}$ - coefficient of variation.

\begin{tabular}{lllll}
\hline Treatment & EL [\%] & RWC $[\%]$ & Chl $\left[\mathrm{mg} \mathrm{g}^{-1}(\mathrm{FM})\right]$ & Pro $\left[\mu \mathrm{mol} \mathrm{g}{ }^{-1}(\mathrm{FM})\right]$ \\
\hline C & $15.40 \pm 1.63^{\mathrm{f}}$ & $78 \pm 1.43^{\mathrm{bc}}$ & $0.73 \pm 0.081^{\mathrm{ab}}$ & $29.00 \pm 4.89^{\mathrm{f}}$ \\
Put 0.5 & $13.40 \pm 0.81^{\mathrm{fg}}$ & $80 \pm 4.08^{\mathrm{ab}}$ & $0.74 \pm 0.016^{\mathrm{a}}$ & $21.14 \pm 2.44^{\mathrm{ghi}}$ \\
Put 1 & $14.50 \pm 1.63^{\mathrm{fg}}$ & $78 \pm 1.63^{\mathrm{bc}}$ & $0.72 \pm 0.015^{\mathrm{ab}}$ & $27.79 \pm 2.04^{\mathrm{f}}$ \\
Spd 0.5 & $12.21 \pm 1.63^{\mathrm{g}}$ & $82 \pm 4.10^{\mathrm{ab}}$ & $0.75 \pm 0.041^{\mathrm{a}}$ & $18.73 \pm 1.63^{\mathrm{i}}$ \\
Spd 1 & $14.30 \pm 1.60^{\mathrm{fg}}$ & $80 \pm 4.08^{\mathrm{ab}}$ & $0.74 \pm 0.032^{\mathrm{a}}$ & $19.97 \pm 0.81^{\mathrm{hi}}$ \\
Spm 0.5 & $12.40 \pm 0.82^{\mathrm{g}}$ & $84 \pm 3.26^{\mathrm{a}}$ & $0.75 \pm 0.088^{\mathrm{a}}$ & $24.48 \pm 4.43^{\mathrm{fgh}}$ \\
Spm 1 & $14.70 \pm 0.86 \mathrm{f}^{\mathrm{g}}$ & $78 \pm 1.63^{\mathrm{bc}}$ & $0.76 \pm 0.081^{\mathrm{a}}$ & $25.98 \pm 4.08^{\mathrm{fg}}$ \\
S & $42.70 \pm 3.26^{\mathrm{a}}$ & $55 \pm 4.08^{\mathrm{h}}$ & $0.42 \pm 0.016^{\mathrm{f}}$ & $81.00 \pm 5.71^{\mathrm{a}}$ \\
S + Put 0.5 & $33.37 \pm 2.57^{\mathrm{bc}}$ & $65 \pm 4.1^{\text {efg }}$ & $0.56 \pm 0.048^{\mathrm{de}}$ & $45.22 \pm 4.08^{\mathrm{d}}$ \\
S + Put 1 & $35.40 \pm 4.08^{\mathrm{b}}$ & $60 \pm 4.08^{\mathrm{gh}}$ & $0.50 \pm 0.081^{\mathrm{ef}}$ & $75.22 \pm 4.10^{\mathrm{b}}$ \\
S + Spd 0.5 & $24.20 \pm 1.63^{\mathrm{e}}$ & $74 \pm 3.26^{\mathrm{cd}}$ & $0.65 \pm 0.041^{\mathrm{bc}}$ & $38.84 \pm 1.63^{\mathrm{e}}$ \\
S + Spd 1 & $26.30 \pm 1.53^{\mathrm{e}}$ & $69 \pm 7.34^{\mathrm{de}}$ & $0.60 \pm 0.082^{\mathrm{cd}}$ & $40.17 \pm 3.26^{\mathrm{de}}$ \\
S + Spm 0.5 & $29.95 \pm 1.25^{\mathrm{d}}$ & $66 \pm 4.89^{\mathrm{ef}}$ & $0.58 \pm 0.065^{\mathrm{cde}}$ & $42.24 \pm 4.85^{\mathrm{de}}$ \\
S + Spm 1 & $32.35 \pm 0.90^{\mathrm{cd}}$ & $61 \pm 0.82^{\mathrm{fg}}$ & $0.56 \pm 0.048^{\mathrm{de}}$ & $60.63 \pm 4.08^{\mathrm{c}}$ \\
ANOVA & & & & \\
Treatment & $* * *$ & $* * *$ & $* *$ & 10.6 \\
CV [\%] & 14.7 & 13.8 & 12.3 & \\
\hline
\end{tabular}

starch content, SOD and CAT activity of Bakraii leaves were significantly influenced by the salt stress and PAs application (Table 4). The maximum amount of protein was observed after the Spd treatment $(0.5 \mathrm{mM})$ under $75 \mathrm{mM} \mathrm{NaCl}$, while the lowest protein content was recorded for PA-nontreated seedlings in $75 \mathrm{mM} \mathrm{NaCl}$ (Table 4). There was significant difference in starch content between control and salinity treatment. The minimum starch content was recorded for seedlings without PAs under the $75 \mathrm{mM} \mathrm{NaCl}$. SOD and CAT activity increased significantly in leaves with increasing the salinity (Table 4). Application of PAs significantly increased the activity of the enzymes (Table 4). Among the PAs, Spd caused the highest activity of SOD and CAT at $75 \mathrm{mM} \mathrm{NaCl}$. The minimum activity of SOD and CAT was related to PA-nontreated seedlings without salinity (Table 4). Among the PAs, the most pronounced difference was found for Spd-treated seedlings and the highest activity was recorded for the seedlings treated with Spd (0.5 $\mathrm{mM})$ at $75 \mathrm{mM} \mathrm{NaCl}$ (Table 4). 
Table 4. Protein, starch, antioxidant enzyme activities in leaves of Iranian mandarin Bakraii seedlings treated with PAs and $\mathrm{NaCl}$. Each value was mean $\pm \mathrm{SD}(n=5)$. Mean values followed by the same letters within a column are not significantly different at $p<0.05$. Analysis of variance $(A N O V A)$; ${ }^{* * *}$ - significant $(P<0.001)$; ${ }^{*}$ - significant $(P<0.01)$. C - control; CAT - catalase; PAs - polyamine; Put - putrescine; S - $75 \mathrm{mM} \mathrm{NaCl}$; SOD - superoxide dismutase; Spd - spermidine; Spm - spermine; CV - coefficient of variation.

\begin{tabular}{lllll}
\hline Treatment & $\begin{array}{l}\text { Protein } \\
{\left[\mathrm{mg} \mathrm{g}^{-1}(\mathrm{FM})\right]}\end{array}$ & $\begin{array}{l}\text { Starch } \\
{\left[\mathrm{mg} \mathrm{g}^{-1}(\mathrm{FM})\right]}\end{array}$ & $\begin{array}{l}\mathrm{SOD} \\
{\left[\mathrm{U} \mathrm{mg}^{-1}(\text { protein })\right]}\end{array}$ & $\begin{array}{l}\text { CAT } \\
{\left[\mu \mathrm{mol} \mathrm{H} \mathrm{O}_{2} \mathrm{mg}^{-1}(\text { protein }) \mathrm{min}^{-1}\right]}\end{array}$ \\
\hline C & $2.73 \pm 0.24^{\mathrm{c}}$ & $76.60 \pm 3.26^{\mathrm{b}}$ & $11.00 \pm 0.41^{\mathrm{j}}$ & $0.07 \pm 0.02^{\mathrm{h}}$ \\
Put 0.5 & $2.80 \pm 0.08^{\mathrm{c}}$ & $87.60 \pm 2.44^{\mathrm{a}}$ & $15.12 \pm 0.40^{\mathrm{h}}$ & $0.09 \pm 0.01^{\mathrm{gh}}$ \\
Put 1 & $2.77 \pm 0.41^{\mathrm{c}}$ & $57.80 \pm 2.45^{\mathrm{efg}}$ & $13.80 \pm 0.41^{\mathrm{i}}$ & $0.14 \pm 0.02^{\mathrm{e}}$ \\
Spd 0.5 & $2.86 \pm 0.41^{\mathrm{bc}}$ & $85.30 \pm 4.08^{\mathrm{a}}$ & $17.9 \pm 0.31^{\mathrm{f}}$ & $0.11 \pm 0.02^{\mathrm{efg}}$ \\
Spd 1 & $2.81 \pm 0.16^{\mathrm{bc}}$ & $58.60 \pm 1.63^{\mathrm{efg}}$ & $16.5 \pm 0.42^{\mathrm{g}}$ & $0.13 \pm 0.08^{\mathrm{ef}}$ \\
Spm 0.5 & $2.77 \pm 0.45^{\mathrm{c}}$ & $67.50 \pm 2.44^{\mathrm{c}}$ & $16.5 \pm 0.32^{\mathrm{g}}$ & $0.11 \pm 0.02^{\mathrm{efg}}$ \\
Spm 1 & $2.73 \pm 0.16^{\mathrm{c}}$ & $65.50 \pm 4.08^{\mathrm{cd}}$ & $15.12 \pm 0.81^{\mathrm{h}}$ & $0.10 \pm 0.02^{\mathrm{fgh}}$ \\
S & $2.06 \pm 0.16^{\mathrm{d}}$ & $22.70 \pm 1.63^{\mathrm{h}}$ & $19.00 \pm 0.81^{\mathrm{e}}$ & $0.30 \pm 0.08^{\mathrm{d}}$ \\
S + Put 0.5 & $3.16 \pm 0.21^{\mathrm{b}}$ & $60.20 \pm 4.08^{\mathrm{ef}}$ & $22.45 \pm 0.81^{\mathrm{cd}}$ & $0.35 \pm 0.02^{\mathrm{bc}}$ \\
S + Put 1 & $2.24 \pm 0.17^{\mathrm{d}}$ & $55.20 \pm 4.18^{\mathrm{g}}$ & $19.90 \pm 0.72^{\mathrm{e}}$ & $0.33 \pm 0.03^{\mathrm{cd}}$ \\
S + Spd 0.5 & $3.74 \pm 0.24^{\mathrm{a}}$ & $66.30 \pm 3.26^{\mathrm{cd}}$ & $25.90 \pm 0.81^{\mathrm{a}}$ & $0.44 \pm 0.02^{\mathrm{a}}$ \\
S + Spd 1 & $2.23 \pm 0.16^{\mathrm{d}}$ & $62.00 \pm 1.63^{\mathrm{de}}$ & $24.20 \pm 0.61^{\mathrm{b}}$ & $0.42 \pm 0.01^{\mathrm{a}}$ \\
S + Spm 0.5 & $2.81 \pm 0.18^{\mathrm{bc}}$ & $58.20 \pm 1.06^{\mathrm{efg}}$ & $23.30 \pm 0.81^{\mathrm{bc}}$ & $0.37 \pm 0.02^{\mathrm{b}}$ \\
S + Spm 1 & $2.69 \pm 0.16^{\mathrm{c}}$ & $56.30 \pm 3.26^{\mathrm{fg}}$ & $21.60 \pm .0 .76^{\mathrm{d}}$ & $0.36 \pm 0.02^{\mathrm{bc}}$ \\
ANOVA & & & & \\
Treatment & $* * *$ & $* * *$ & $* *$ & 12.6 \\
CV [\%] & 7.4 & 6.2 & 9.5 & \\
\hline
\end{tabular}

Table 5. Gas-exchange characteristics in leaves of Iranian mandarin Bakraii seedlings treated with PAs and NaCl. Each value was mean $\pm \mathrm{SD}(n=5)$. Mean values followed by the same letters within a column are not significantly different at $p<0.05$. Analysis of variance (ANOVA); ${ }^{* * *}$ - significant $(P<0.001) ; * *$ - significant $(P<0.01) . \mathrm{C}-$ control; $C_{\mathrm{i}}$ - intercellular $\mathrm{CO}_{2}$ concentration; $g_{\mathrm{s}}-$ stomatal conductance; PAs - polyamine; $P_{\mathrm{N}}$ - net photosynthetic rate; Put - putrescine; $\mathrm{S}$ - $75 \mathrm{mM} \mathrm{NaCl}$; Spd - spermidine; Spm - spermine; $\mathrm{CV}$ - coefficient of variation.

\begin{tabular}{llll}
\hline Treatment & $\begin{array}{l}P_{\mathrm{N}} \\
{\left[\mu \mathrm{mol}\left(\mathrm{CO}_{2}\right) \mathrm{m}^{-2} \mathrm{~s}^{-1}\right]}\end{array}$ & $\begin{array}{l}g_{\mathrm{s}} \\
{\left[\mathrm{mmol}\left(\mathrm{H}_{2} \mathrm{O}\right) \mathrm{m}^{-2} \mathrm{~s}^{-1}\right]}\end{array}$ & $\begin{array}{l}C_{\mathrm{i}} \\
{\left[\mu \mathrm{mol} \mathrm{mol}{ }^{-1}\right]}\end{array}$ \\
\hline $\mathrm{C}$ & $6.2 \pm 0.16^{\mathrm{b}}$ & $0.34 \pm 0.02^{\mathrm{ab}}$ & $152 \pm 2.45^{\mathrm{bc}}$ \\
Put 0.5 & $6.4 \pm 0.32^{\mathrm{ab}}$ & $0.32 \pm 0.01^{\mathrm{abc}}$ & $158 \pm 1.63^{\mathrm{a}}$ \\
Put 1 & $6.2 \pm 0.16^{\mathrm{b}}$ & $0.30 \pm 0.02^{\mathrm{cd}}$ & $153 \pm 2.44^{\mathrm{abc}}$ \\
Spd 0.5 & $6.4 \pm 0.16^{\mathrm{ab}}$ & $0.33 \pm 0.01^{\mathrm{abc}}$ & $154 \pm 3.26^{\mathrm{abc}}$ \\
Spd 1 & $6.2 \pm 0.26^{\mathrm{b}}$ & $0.30 \pm 0.04^{\mathrm{cd}}$ & $152 \pm 4.08^{\mathrm{bc}}$ \\
Spm 0.5 & $6.6 \pm 0.13^{\mathrm{a}}$ & $0.35 \pm 0.04^{\mathrm{a}}$ & $150 \pm 4.10^{\mathrm{c}}$ \\
Spm 1 & $6.6 \pm 0.16^{\mathrm{a}}$ & $0.31 \pm 0.01^{\mathrm{bcd}}$ & $156 \pm 3.26^{\mathrm{ab}}$ \\
$\mathrm{S}$ & $2.6 \pm 0.48^{\mathrm{g}}$ & $0.15 \pm 0.04^{\mathrm{h}}$ & $119 \pm 0.82^{\mathrm{f}}$ \\
$\mathrm{S}+$ Put 0.5 & $4.0 \pm 0.41^{\mathrm{de}}$ & $0.22 \pm 0.02^{\mathrm{g}}$ & $130 \pm 8.16^{\mathrm{e}}$ \\
S + Put 1 & $3.4 \pm 0.16^{\mathrm{f}}$ & $0.23 \pm 0.02^{\mathrm{fg}}$ & $137 \pm 2.44^{\mathrm{d}}$ \\
$\mathrm{S}+$ Spd 0.5 & $4.8 \pm 0.41^{\mathrm{c}}$ & $0.28 \pm 0.02^{\mathrm{de}}$ & $139 \pm 0.82^{\mathrm{d}}$ \\
S + Spd 1 & $3.7 \pm 0.41^{\mathrm{ef}}$ & $0.26 \pm 0.01^{\mathrm{ef}}$ & $138 \pm 1.63^{\mathrm{d}}$ \\
S + Spm 0.5 & $4.2 \pm 0.17^{\mathrm{d}}$ & $0.25 \pm 0.04^{\mathrm{efg}}$ & $142 \pm 8.16^{\mathrm{d}}$ \\
S + Spm 1 & $3.5 \pm 0.82^{\mathrm{f}}$ & $0.23 \pm 0.03^{\mathrm{fg}}$ & $140 \pm 4.08^{\mathrm{d}}$ \\
ANOVA & & & \\
Treatment & $* * *$ & $* *$ & $* *$ \\
CV [\%] & 9.9 & 8.2 & \\
\hline
\end{tabular}

Photosynthetic parameters: Salinity stress led to a significant decline in $P_{\mathrm{N}}, g_{\mathrm{s}}$, and $C_{\mathrm{i}}$ (Table 5). Application of PAs especially at $0.5 \mathrm{mM}$ Spd significantly increased $P_{\mathrm{N}}, g_{\mathrm{s}}$, and $C_{\mathrm{i}}$ compared with the controls (Table 5). Plants treated with PAs maintained higher $P_{\mathrm{N}}, g_{\mathrm{s}}$, and $C_{\mathrm{i}}$ when subjected to salinity stress. The $\mathrm{F}_{\mathrm{v}} / \mathrm{F}_{\mathrm{m}}$ was significantly affected by salt stress and PAs (Table 6). Salinity stress decreased $F_{v} / F_{m}$ and $F_{v}$ in the leaves of Bakraii seedlings (Tables 6, 7). The $\mathrm{F}_{\mathrm{v}} / \mathrm{F}_{\mathrm{m}}$ in the dark-adapted state significantly decreased due to the salt-induced increase of $\mathrm{F}_{0}$ and the marked decline of $\mathrm{F}_{\mathrm{m}}$ (Table 6). Salt stress significantly decreased $\mathrm{q}_{\mathrm{P}}$ in the light-adapted state, but markedly elevated NPQ (Table 7). These results indicate harmful effects of $\mathrm{NaCl}$ on metabolic processes ultimately resulting in reduced efficiency of PSII. PAs treatment significantly improved the Chl fluorescence parameters in Bakraii leaves (Tables 6, 7). 
Table 6. Fluorescence parameters, $F_{0}, F_{m}$ and $F_{v} / F_{m}$ in Iranian mandarin Bakraii seedlings treated with PAs and $\mathrm{NaCl}$. Each value was mean $\pm \mathrm{SD}(n=5)$. Mean values followed by the same letters within a column are not significantly different at $p<0.05$. Analysis of variance $($ ANOVA $) ; * * *-\operatorname{significant}(P<0.001) ;{ }^{* *}-$ significant $(P<0.01)$. $\mathrm{C}$ - control; $\mathrm{F}_{\mathrm{m}}$ - maximal fluorescence yield of the dark-adapted state; Fo - minimal fluorescence yield of the dark-adapted state; $\mathrm{F}_{\mathrm{v}} / \mathrm{F}_{\mathrm{m}}$ - maximum photochemical efficiency of PSII; PAs - polyamine; Put - putrescine; S - $75 \mathrm{mM}$ $\mathrm{NaCl}$; Spd - spermidine; Spm - spermine; CV - coefficient of variation.

\begin{tabular}{llll}
\hline Treatment & $\mathrm{F}_{0}$ & $\mathrm{~F}_{\mathrm{m}}$ & $\mathrm{F}_{\mathrm{v}} / \mathrm{F}_{\mathrm{m}}$ \\
\hline $\mathrm{C}$ & $402 \pm 16.32^{\mathrm{f}}$ & $2,070 \pm 1.63^{\mathrm{a}}$ & $0.81 \pm 0.01^{\mathrm{a}}$ \\
Put 0.5 & $410 \pm 8.16^{\mathrm{ef}}$ & $2,079 \pm 8.16^{\mathrm{a}}$ & $0.80 \pm 0.04^{\mathrm{a}}$ \\
Put 1 & $418 \pm 1.63^{\mathrm{de}}$ & $1,980 \pm 16.32^{\mathrm{c}}$ & $0.79 \pm 0.02^{\mathrm{a}}$ \\
Spd 0.5 & $410 \pm 8.16^{\mathrm{ef}}$ & $2,079 \pm 0.82^{\mathrm{a}}$ & $0.80 \pm 0.01^{\mathrm{a}}$ \\
Spd 1 & $430 \pm 8.16^{\mathrm{d}}$ & $2,002 \pm 1.63^{\mathrm{b}}$ & $0.78 \pm 0.02^{\mathrm{a}}$ \\
Spm 0.5 & $400 \pm 16.32^{\mathrm{f}}$ & $1,970 \pm 24.49^{\mathrm{c}}$ & $0.79 \pm 0.01^{\mathrm{a}}$ \\
Spm 1 & $420 \pm 16.32^{\mathrm{de}}$ & $1,850 \pm 40.82^{\mathrm{d}}$ & $0.77 \pm 0.02^{\mathrm{ab}}$ \\
S & $510 \pm 8.16^{\mathrm{a}}$ & $1,039 \pm 0.82^{\mathrm{k}}$ & $0.51 \pm 0.08^{\mathrm{g}}$ \\
S + Put 0.5 & $480 \pm 8.20^{\mathrm{bc}}$ & $1,400 \pm 16.32^{\mathrm{h}}$ & $0.65 \pm 0.04^{\mathrm{de}}$ \\
S + Put 1 & $490 \pm 7.30^{\mathrm{b}}$ & $1,210 \pm 8.16^{\mathrm{j}}$ & $0.59 \pm 0.01^{\mathrm{f}}$ \\
S + Spd 0.5 & $474 \pm 5.40^{\mathrm{c}}$ & $1,809 \pm 7.34^{\mathrm{e}}$ & $0.72 \pm 0.02^{\mathrm{bc}}$ \\
S + Spd 1 & $484 \pm 8.10^{\mathrm{bc}}$ & $1,545 \pm 4.08^{\mathrm{f}}$ & $0.68 \pm 0.02^{\mathrm{cd}}$ \\
S + Spm 0.5 & $510 \pm 7.16^{\mathrm{a}}$ & $1,455 \pm 4.08^{\mathrm{g}}$ & $0.65 \pm 0.04^{\mathrm{de}}$ \\
S + Spm 1 & $489 \pm 2.45^{\mathrm{b}}$ & $1,273 \pm 8.16^{\mathrm{i}}$ & $0.60 \pm 0.04^{\mathrm{ef}}$ \\
ANOVA & & & \\
Treatment & $* * *$ & $* * *$ & $* *$ \\
CV [\%] & 15.4 & 4.8 & 9.3 \\
\hline
\end{tabular}

\section{Discussion}

PAs have various functions in all living cells, but the precise nature of their impact under stress conditions still remains a matter of debate. $\mathrm{NaCl}$ stress led to reduction of growth parameters in Bakraii seedlings. Similar results were reported by numerous investigators (García-Sánchez et al. 2002). Can et al. (2003) reported that irrigation plants with saline water reduced the photosynthetic capacity per unit of area as well as further depressed vegetative growth of the whole tree. Besides, Cooper and Gorton (1952) mentioned that the decrement in the growth of the plants grown under salinity conditions might be attributed to an increase in the osmotic potential of the soil solution causing a marked depression in the water-absorbing power of the plants. This study demonstrated that the exogenous application of PAs decreased the severity of the stress injury in Bakraii plants. Foliar-applied PAs due to their ability to act as growth regulators are able to modulate the plant metabolism and the production of metabolites involved in stress tolerance (Takahama and Oniki 1997). PAs are involved in plant defense to environmental stresses (Bouchereau et al. 1999). It has been suggested that the main role of PAs is to maintain a cation-anion balance during long-term salinity (Santa-Cruz et al. 1997). Hence, the use of exogenous PAs increased the physiological characteristics by increasing growth (Parvin et al. 2014). The individual PAs may play different roles
Table 7. $F_{v}, q_{P}$ and NPQ in Iranian mandarin Bakraii seedlings treated with PAs and $\mathrm{NaCl}$. Each value was mean $\pm \mathrm{SD}(n=5)$. Mean values followed by the same letters within a column are not significantly different at $p<0.05$. Analysis of variance (ANOVA); *** - significant $(P<0.001) ; * *$ - significant $(P<0.01)$. $\mathrm{C}$ - control; $\mathrm{F}_{\mathrm{v}}$ - variable fluorescence; NPQ - nonphotochemical quenching; PAs - polyamine; Put - putrescine; qP photochemical quenching; $\mathrm{S}-75 \mathrm{mM} \mathrm{NaCl}$; $\mathrm{Spd}$ - spermidine; $\mathrm{Spm}$ - spermine; CV - coefficient of variation.

\begin{tabular}{llll}
\hline Treatment & $\mathrm{F}_{\mathrm{v}}$ & $\mathrm{q}_{\mathrm{p}}$ & $\mathrm{NPQ}$ \\
\hline C & $1,668 \pm 8.16^{\mathrm{a}}$ & $0.75 \pm 0.04^{\mathrm{a}}$ & $0.67 \pm 0.02^{\text {ef }}$ \\
Put 0.5 & $1,669 \pm 1.63^{\mathrm{a}}$ & $0.77 \pm 0.02^{\mathrm{a}}$ & $0.62 \pm 0.02^{\mathrm{gh}}$ \\
Put 1 & $1,562 \pm 1.63^{\mathrm{b}}$ & $0.76 \pm 0.05^{\mathrm{a}}$ & $0.70 \pm 0.04^{\mathrm{de}}$ \\
Spd 0.5 & $1,669 \pm 1.63^{\mathrm{a}}$ & $0.78 \pm 0.02^{\mathrm{a}}$ & $0.65 \pm 0.04^{\mathrm{fg}}$ \\
Spd 1 & $1,572 \pm 8.16^{\mathrm{b}}$ & $0.77 \pm 0.03^{\mathrm{a}}$ & $0.60 \pm 0.05^{\mathrm{h}}$ \\
Spm 0.5 & $1,570 \pm 8.20^{\mathrm{b}}$ & $0.77 \pm 0.04^{\mathrm{a}}$ & $0.67 \pm 0.03^{\text {ef }}$ \\
Spm 1 & $1,430 \pm 24.49^{\mathrm{c}}$ & $0.75 \pm 0.03^{\mathrm{a}}$ & $0.68 \pm 0.01^{\text {ef }}$ \\
S & $529 \pm 8.16^{\mathrm{g}}$ & $0.50 \pm 0.08^{\mathrm{e}}$ & $0.98 \pm 0.02^{\mathrm{a}}$ \\
S + Put 0.5 & $920 \pm 16.32^{\mathrm{e}}$ & $0.59 \pm 0.01^{\mathrm{d}}$ & $0.78 \pm 0.03^{\mathrm{c}}$ \\
S + Put 1 & $720 \pm 16.22^{\mathrm{f}}$ & $0.57 \pm 0.02^{\mathrm{d}}$ & $0.85 \pm 0.02^{\mathrm{b}}$ \\
S + Spd 0.5 & $1,335 \pm 24.49^{\mathrm{c}}$ & $0.68 \pm 0.01^{\mathrm{b}}$ & $0.69 \pm 0.01^{\text {de }}$ \\
S + Spd 1 & $1,061 \pm 16.32^{\mathrm{d}}$ & $0.65 \pm 0.04^{\mathrm{bc}}$ & $0.78 \pm 0.01^{\mathrm{c}}$ \\
S + Spm 0.5 & $945 \pm 16.32^{\mathrm{e}}$ & $0.62 \pm 0.02^{\mathrm{cd}}$ & $0.72 \pm 0.04^{\mathrm{d}}$ \\
S + Spm 1 & $784 \pm 8.16^{\mathrm{f}}$ & $0.60 \pm 0.08^{\mathrm{cd}}$ & $0.80 \pm 0.01^{\mathrm{c}}$ \\
ANOVA & & & \\
Treatment & $* *$ & $* *$ & $* * *$ \\
CV [\%] & 7.4 & 8.4 & 7.3 \\
\hline & & &
\end{tabular}

during the plant response to salt stress. These differences may result from the differences in concentration of the PAs used and vary according to species and even cultivars of a species (Tonon et al. 2004).

The presence of $\mathrm{NaCl}$ in the water used for irrigation or in soil changes the nutritional balance of plants, leading to high ratios of $\mathrm{Na}^{+} / \mathrm{Ca}^{+2}, \mathrm{Na}^{+} / \mathrm{Mg}^{+2}$, and $\mathrm{Na}^{+} / \mathrm{K}^{+}$that can cause the inhibition of the growth and yield in citrus crops. The main saline ions, $\mathrm{Na}^{+}$and $\mathrm{Cl}^{-}$, can influence the nutrient absorption through competitive interactions or by influencing the ion selectivity of membranes (Behboudian et al. 1986). High concentration of $\mathrm{Na}^{+}$and $\mathrm{Cl}^{-}$in leaves also causes substantial reduction in $g_{\mathrm{s}}$ and $P_{\mathrm{N}}$ in citrus (García-Sánchez et al. 2002). In the present study, $\mathrm{K}^{+}$ concentration in leaves increased at $75 \mathrm{mM} \mathrm{NaCl}$. Other researchers have reported increased amount of potassium under salt conditions (Behboudian et al. 1986). This might occur to balance the excess $\mathrm{Cl}^{-}$in leaf cells to ensure the viability of the leaves, since $\mathrm{K}^{+}$is the most abundant and highly mobile (in both xylem and phloem) cation in plants (Marschner 1995). It seems that one of the mechanisms to be tolerant under the average salinity increased $\mathrm{K}^{+}$ concentration in leaves of citrus. According to the results obtained in this experiment, we determined that with increasing salinity, the amount of $\mathrm{Ca}^{2+}$ in leaves decreased. Reduction in the amount of $\mathrm{Ca}^{2+}$ in the shoots under salt 
stress can be due to the competitive effects of ions in vessels of the plant with calcium, which disrupt the transfer of this element (Botella et al. 1997). $\mathrm{Ca}^{2+}$ is important in cell biology (especially in membrane biology) during salt stress, e.g., in preserving membrane integrity (Rengel 1992), signaling in osmoregulation (Mansfield et al. 1990), and influencing $\mathrm{K}^{+} / \mathrm{Na}^{+}$selectivity (Cramer et al. 1987). $\mathrm{Mg}^{2+}$ decreased in leaves of Bakraii as salt concentration increased. Reports of Ruiz et al. (1997) and García-Sánchez (2002) show reduced contents of $\mathrm{Mg}^{2+}$ in shoots of different citrus cultivars under salt stress which is consistent with the results of this experiment. Very little is known about salinity and $\mathrm{Mg}^{2+}$ interactions in citrus. However, besides its role in Chl structure and as an enzyme cofactor, another important role of $\mathrm{Mg}^{2+}$ in plants is in photosynthesis, which is impaired and leads to enhanced degradation of $\mathrm{Chl}$ in $\mathrm{Mg}^{2+}$-deficient source leaves, resulting in increased oxygenase activity of Rubisco (Marschner and Cakmak 1989). In $\mathrm{Mg}^{2+}$-deficient leaves, formation of superoxide radicals and hydrogen peroxide is enhanced and therefore the leaves become highly photosensitive. $\mathrm{Mg}^{2+}$ deficiency may be one of the reasons for the chlorosis observed in $\mathrm{NaCl}$-treated plants (Tozlu et al. 2000). Foliar application of PAs to salt-treated seedlings resulted in reduced $\mathrm{Na}^{+}$and $\mathrm{Cl}^{-}$accumulation in leaves. Under PAs treatmets, plant $\mathrm{K}^{+} / \mathrm{Na}^{+}$ratio is affected significantly. Maintaining $\mathrm{K}^{+} / \mathrm{Na}^{+}$is an important mechanism for plants to adapt to salt tolerance. Our studies suggested that exogenous PAs could significantly alleviate the inhibition of growth in citrus seedlings subjected to $\mathrm{NaCl}$ stress and markedly improve the plant growth. Due to their polycationic nature, PAs, especially Spd, contribute to maintaining cellular cation-anion balance, and may act as a signaling regulator responsible for inward rectification of rectifying $\mathrm{K}^{+}$channels and certain $\mathrm{Ca}^{2+}$ permeable channels (Yamaguchi et al. 2007, Roy et al. 2005, Smith 1985). Consequently, exogenous PAs pretreatment might promote salinity tolerance by regulating the ion metabolism in Bakraii.

An increase in electrical conductivity indicates elevated leakiness of ions due to a loss of membrane integrity. This is an inherent feature of plants which are exposed to stresses such as salinity (Sharma et al. 2011). This study showed that PAs reduced the ion leakage (measured as electrolytes) in salt-stressed Bakraii plants indicating that PAs treatment facilitated the maintenance of membrane functions under stress conditions. Maintaining integrity of cellular membranes under salt stress is considered an integral part of salinity-tolerance mechanism (Stevens et al. 2006). This is probably due to the fact that PAs causes enhacement in the activities of antioxidant enzymes, which, in turn, protect plants against the generation of ROS and membrane injury, or may result in the synthesis of other substances, which have a protective effect on plants growing under salt stress (Upchurch 2008). In addition to their properties as free radical scavengers, PAs, particularly bound PAs, were also reported to stabilize biological membrane by direct binding to membrane phospholipids under stress conditions (Todorova et al. 2007). Exogenous Spd prevented the leakage of electrolytes and amino acids and recovered the damage of plasma membrane in rice cultivars in response to salinity (Roy et al. 2005). RWC in leaves is known as an alternative measure of plant water status, reflecting the metabolic activity in tissues (González and González-Vilar 2001). RWC reduced by salinity which was in agreement with Stępień and Kłbus (2006). RWC reduction indicated a loss of turgor that resulted in limited water availability for the cell extension process (Katerji et al. 1997) and may result from lower water availability under stress conditions (Shalhevet 1993), and/or root systems which are not able to compensate for water lost by transpiration (Gadallah 2000). Exogenous application of PAs, especially Spm and Spd, noticeably alleviated the negative effects of salt stress and improved the growth of seedlings. This agrees with the findings that PAs induce tolerance to many abiotic stresses (Bouchereau et al. 1999). For example, exogenously applied PAs have been shown to be beneficial in sustaining growth of different crops under salt stress (Çavuşoğlu et al. 2007).

In this study, salinity stress caused a decrease in a protein content of leaves which confirms the report of Sharma and Dubey (2010), while foliar application of PAs increased the protein content. In plants, PAs, especially Spd and Spm, seem to play an important role via interacting with many anionic molecules, such as DNA, RNA, proteins, and membrane lipids due to their polycationic nature (Bouchereau et al. 1999). Moreover, free radicals, such as $\mathrm{H}_{2} \mathrm{O}_{2}$ accumulating under salt stress, physically distored the structure of proteins, leading to reduction of their content. Therefore, the impact of PAs might be due to either preventing the production of free radicals or free radical-scavenging mechanisms and subsequently protecting the proteins (Peltzer et al. 2002).

In general, under salt stress, starch contents decrease (Chaves et al. 2009), which is in accordance with the present study. Photosynthesis is inhibited under the stress resulting in a reduced pool of starch and soluble carbohydrates within the leaves (Demetriou et al. 2007). The implication of PAs, increasing the starch in leaves under the salt stress, might be due to the constructive role of PAs in improving and maintaining the structure and function of photosynthetic apparatus during salinity stress.

Results showed that under salinity stress, the Pro concentration of Bakraii leaves increased. In this regard, Zhu (2001) reported that plants accumulate compatible osmolytes such as Pro when they are subjected to salinity stress. Pro has multiple functions, such as osmotic pressure regulation, protection of membrane integrity, stabilization of enzymes/proteins, maintaining appropriate $\mathrm{NADP}^{+} /$ NADPH ratios, and scavenging free radicals (Hare and Cress 1997). Accumulation of Pro under stress conditions such as high salinity, in plants, has been correlated with stress tolerance (Misra and Gupta 2005). However, in our 
study, PAs caused a decrease in the content of Pro in leaves showing other mechanisms, by which PAs reduced the impact of salinity stress. In this study, PAs application reduced salt-induced Pro accumulation which suggested that salt stress might be partially alleviated without requiring the accumulation of Pro to high concentrations. This appears to be supported by Jiménez-Bremont et al. (2006) who reported that exogenous PAs decreased Pro accumulation in salinity-exposed Phaseolus vulgaris L. The Pro degradation seems to be beneficial in the response to stress, given that the degradation of proline to glutamate generates reducing equivalents that support mitochondrial oxidative phosphorylation (Hare and Cress 1997). Furthermore, the proline dehydrogenase activity is capable of consuming $\mathrm{O}_{2}$ (Hare and Cress 1997), and perhaps could reduce the oxidizing power of the cell and in turn possibly generate ROS (Rosales et al. 2007). On the other hand, PAs appears to be capable of mitigating damage associated with salinity stress by reducing oxidative stress and inducing Pro degradation, mechanisms that permit the plant to adapt under these conditions.

In this study, antioxidant enzyme activities (SOD and CAT) increased with the salinity. Salinity has been reported to increase SOD activity in pea plants (Hernández et al. 2000) and in citrus (Almansa et al. 2002). CAT is an important antioxidant enzyme, which catabolizes hydrogen peroxide (Larsen et al. 1988). Increasing activities of SOD and CAT with the application of PAs proved the role of these plant growth regulators in mitigating the adverse effects of salt stress in citrus. This finding confirms the report of Parida and Das (2005) in regard to the mutual relationship between higher antioxidant activity and salinity tolerance (Parida and Das 2005). Previous finding proposed that transgenic tobacco may significantly induce the expression of antioxidant enzymes by elevating the PAs content (Wi et al. 2006), resulting in tolerance to different abiotic stresses. It is evident that PAs exhibit their antioxidant effect by inducing the expression of genes encoding antioxidant enzymes. Therefore, PAs may function not only as scavengers of ROS, but also as activators of the expressions of genes encoding antioxidant enzymes (Parvin et al. 2014). $\mathrm{H}_{2} \mathrm{O}_{2}$ is one of the major and most stable ROS and its high concentration leads to oxidative stress through an increase of lipid peroxidation and modification of membrane permeability (Upchurch 2008); PAs minimize oxidative injury in plants by acting as direct free radical scavengers (Bors et al. 1989). Therefore, it seems that one mechanism, in which foliar application of PAs alleviates salt stress in Bakraii seedlings, is to increase SOD and CAT activities. Although previous findings suggested that Put might activate antioxidant enzymes and elevate antioxidants in Indian mustard (Verma and Mishra 2005) and Virginia pine seedlings under salinity stress (Tang and Newton 2005), our results showed a more important role of Spd and Spm compared to Put in alleviating salt stress in Bakraii seedlings. It is well documented that PAs counteract oxidative damage in plants by acting as direct free radical scavengers or binding to antioxidant enzyme molecules to scavenge free radical (Bors et al. 1989).

In the present investigation, $\mathrm{Chl}, P_{\mathrm{N}}, \mathrm{g}_{\mathrm{s}}, C_{\mathrm{i}}, \mathrm{F}_{\mathrm{v}}, \mathrm{F}_{\mathrm{m}}$, $\mathrm{F}_{\mathrm{v}} / \mathrm{F}_{\mathrm{m}}$, and $\mathrm{q}_{\mathrm{p}}$ decreased with increasing salinity. A decrease in leaf $\mathrm{Chl}$ content has been described in citrus rootstocks irrigated with high $\mathrm{NaCl}$ concentration (Koshbakht and Asgharei 2015, García-Sánchez et al. 2002). This loss of Chl content could be associated with accumulation of $\mathrm{Cl}^{-}$and $\mathrm{Na}^{+}$in the leaves (Anjum 2007). The decrease in leaf $\mathrm{Chl}$ concentration could be due to changes in the lipid protein ratio of pigment-protein complexes (Rao and Rao 1981), increased chlorophyllase activity, and degradation (Singh and Dubey 1995) and an inhibition if synthesis of photosynthetic pigments (GarcíaSánchez et al. 2002). Photosynthetic pigments present in the photosystems are believed to be damaged by stress factors resulting in a reduced light-absorbing efficiency of both photosystems (PSI and PSII) and hence a reduced photosynthetic capacity (Zhang et al. 2011). In the present study, salinity-induced reductions in $P_{N}, g_{\mathrm{s}}$, and $C_{i}$ were alleviated by exogenous PAs, in accordance with a similar experiment, implying that exogenous PAs alleviates the damage of photosynthetic apparatus under salinity conditions (Anjum 2009). Hence, the use of exogenous PAs increased the physiological characteristics of Bakraii seedlings by increasing both pigments and growth. The $F_{v} / F_{m}$ is correlated with the efficiency of leaf photosynthesis. A decline in this ratio provides an indicator of photoinhibitory damage caused by the incident photon flux density when plants are subjected to a wide range of environmental stresses (Björkman and Demming 1987). The salt-induced increase of $\mathrm{F}_{0}$ and the marked decline of $F_{m}$ in Bakraii implied the inhibitory effects of salt ions on the potential quantum efficiency of PSII, although the elevated NPQ may serve to dissipate excess excitation energy (Wang et al. 2007). The reduction of $F_{v}$ along an increase in $F_{0}$ is considered to be the characteristic of inhibition of the acceptor side of PSII (Šetlík et al. 1990). The reduction in $F_{v} / F_{m}$ and $q_{p}$ were correlated with an increase in NPQ suggesting that salt treatment induced dissipation of damaging excessive energy. This energy could be dissipated at antennae through heat emission although other mechanisms are also involved in energy dissipation, e.g., related to a transmembrane $\mathrm{H}^{+}$gradient generated by ATPase activity and uncoupling of electron transport, which may lead to oxidative stress (Maxwell and Johnson 2000). Earlier studies have indicated that salt stress reduced $P_{\mathrm{N}}$ and $g_{\mathrm{s}}$ in the leaves of many crops (Syeed et al. 2011). A decline in photosynthetic capacity under salt stress is often associated with the generation of ROS (Noreen et al. 2010). All of these factors ultimately reduce overall growth of plants. Salt stress hinders $P_{\mathrm{N}}$ at multiple levels, such as pigments, stomatal functioning, gaseous exchange, structure and function of thylakoid membrane, electron transport, and enzyme activities, by hampering the oxidative stress mitigation mechanisms and cellular 
metabolism of plants (Sudhir and Murthy 2004). Excessive salt concentrations might cause the closure of stomata, thereby decreasing the partial $\mathrm{CO}_{2}$ pressure and $C_{\mathrm{i}}$ and consequently resulting in a decreased $P_{\mathrm{N}}$ (Bethke and Drew 1992). It is well documented that PAs are involved in protection against different environmental stresses in plants (Bouchereau et al. 1999). PAs exert positive effects on photosynthetic efficiency under stress conditions due to their acid-neutralizing and antioxidant properties, as well as their membrane- and cell wall-stabilizing activity (Mapelli et al. 2008). PAs with a high net positive charge can stabilize PSII proteins, such as D1 and D2, under photoinhibition conditions (Hamdani et al. 2011). PAs binding to membrane proteins may stabilize the protein structure during stress and consequently preserve photosynthetic activity (Hamdani et al. 2011). There have been several reports establishing that PAs, especially the thylakoid-bound PAs, participate in the regulation of structure and function of photosynthetic apparatus under

\section{References}

Alcazar R., Altabella T., Marco F. et al.: Polyamines: molecules with regulatory functions in plant abiotic stress tolerance. Planta 231: 1237-1249, 2010.

Almansa M.S., Hernandez J.A., Jimenez A. et al.: Effect of salt stress on the superoxide dismutase activity in leaves of Citrus limonum in different rootstock-scion combinations. - Biol. Plantarum 45: 545-549, 2002.

Anjum M.A.: Effect of exogenously applied spermidine on growth and physiology of citrus rootstock Troyer citrange under saline conditions. - Turk. J. Agric. For. 35: 43-55, 2009.

Anjum M.A.: Effect of $\mathrm{NaCl}$ concentration in irrigation water on growth and polyamine metabolism in two citrus rootstocks with different levels of salinity tolerance. - Acta Physiol. Plant. 30: 43-52, 2008.

Barrs H.D., Weatherley P.E.: A re-examination of the relative turgidity technique for estimating water deficits in leaves. Aust. J. Biol. Sci. 24: 519-570, 1962.

Bates L., Waldren P.P., Teare J.D.: Rapid determination of the free proline of water stress studies. - Plant Soil 39: 205-207, 1973.

Behboudian M.H., Törökfalvy E., Walker R.R.: Effects of salinity on ionic content, water relations and gas exchanges parameters in some citrus scion-rootstock combinations. - Sci. Hortic.-Amsterdam 28: 105-116, 1986.

Bethke P.C., Drew M.C.: Stomatal and non-stomatal components to inhibition of photosynthesis in leaves of Capsicum annuum during progressive exposure to $\mathrm{NaCl}$ salinity. - Plant Physiol. 99: 219-226, 1992.

Bilger W., Johnsen T., Schreiber U.: UV-excited chlorophyll fluorescence as a tool for the assessment of UV-protection by the epidermis of plants. - J. Exp. Bot. 52: 2007-2014, 2001.

Björkman O., Demming B.: Photon yield of oxygen evolution and chlorophyll fluorescence characteristics at $77 \mathrm{~K}$ among vascular plants of diverse origin. - Planta 170: 489-504, 1987.

Bors W., Langebartels C., Michel C. et al.: Polyamines as radical scavengers and protectants against ozone damage. Phytochemistry 28: 1589-1595, 1989.

Botella M.A., Martinez V., Pardines J. et al.: Salinity induced environmental conditions, such as UV-B radiation (Lütz et al. 2005), low temperature (Sfakianaki et al. 2006), and salinity (Lütz et al. 2005, Demetriou et al. 2007).

Conclusion: In summary, this investigation revealed that salt stress had inhibitory effects on growth, gas-exchange characteristics, and Chl fluorescence of Bakraii seedlings. The results suggested the potential for PAs as a potent antioxidant in plants. PAs action might be, at least in part, explained by their ability to (1) increase the activities of SOD and CAT, (2) reduce the uptake of the toxic $\mathrm{Na}^{+}$and $\mathrm{Cl}^{-}$and maintain ionic and osmotic balance, (3) scavenge ROS, to protect chlorophyll content, photosynthesis parameters, and improve photosystem functioning. PAs treatments could ameliorate the negative effect of salinity on the growth of Bakraii and PAs could be used as potential growth regulators to improve plant salinity tolerance. Spd applied at $0.50 \mathrm{mM}$ was the most effective $\mathrm{PA}$ in providing Bakraii seedlings with salt tolerance.

potassium deficiency in maize plant. - Plant Physiol. 150: 200205, 1997.

Bouchereau A., Aziz A., Larher F. et al.: Polyamines and environmental challenges: recent development. - Plant Sci. 140: 103-125, 1999.

Bradford M.M.: A rapid and sensitive method for the quantitation of microgram quantities of protein utilizing the principles of protein-dye binding. - Anal Biochem. 72: 248-254, 1976.

Cakmak I., Marschner H.: Magnesium deficiency and high light intensity enhance activities of superoxide dismutase, ascorbate peroxidase, and glutathione reductase in bean leaves. - Plant Physiol. 98: 1222-1227, 1992.

Can H.Z., Anac D., Kukul Y. et al.: Alleviation of salinity stress by using potassium fertilization in Satsuma mandarin trees budded on two different rootstocks. - Acta Hortic. 618: 275280, 2003.

Çavuşoğlu A, Kiliç S., Kabar K.: Some morphological and anatomical observations during alleviation of salinity $(\mathrm{NaCl})$ stress on seed germination and seedling growth of barley by polyamines. - Acta Physiol. Plant. 29: 551-557, 2007.

Chattopadhayay M.K., Tabor C.W., Tabor H.:. Polyamines protect Escherichia coli cells from the toxic effect of oxygen. - P. Natl. Acad. Sci. USA 100: 2261-2265, 2003.

Chaves M.M., Flexas J., Pinheiro C.: Photosynthesis under drought and salt stress: regulation mechanisms from whole plant to cell. - Ann. Bot.-London 103: 551-560, 2009.

Cooper W.C., Gorton B.S.: Toxicity and accumulation of chloride salts in citrus on various rootstocks. - P. Am. Soc. Hortic. Sci. 59: 143-146, 1952.

Cramer G.R., Lynch J., Läuchli A. et al.: Influx of $\mathrm{Na}^{+}, \mathrm{K}^{+}$, and $\mathrm{Ca}^{2+}$, into roots of salt-stressed cotton seedlings. Effects of supplemental $\mathrm{Ca}^{2+}$. - Plant Physiol. 83: 510-516, 1987.

Demetriou G., Neonaki C., Navakoudis E. et al.: Salt stress impact on the molecular structure and function of the photosynthetic apparatus-the protective role of polyamines. BBA-Bioenergetics 1767: 272-280, 2007.

Dhindsa R.S., Plumb-Dhindsa P., Thorpe T.A.: Leaf senescence: correlated with increased levels of membrane permeability and 
lipid peroxidation, and decreased levels of superoxide dismutase and catalase. - J. Exp. Bot. 32: 93-101, 1981.

Gadallah M.A.A.: Effects of indole-3-acetic acid and zinc on the growth, osmotic potential and soluble carbon and nitrogen components of soybean plants growing under water deficit. - J. Arid. Environ. 44: 451-467, 2000.

Galston A.W., Kaur-Sawhney R., Altabella T. et al.: Plant polyamines in reproductive activity and response to a biotic stress. - Bot. Acta 110: 197-207, 1997.

García-Sánchez F., Carvajal M., Sanchez-Pina M.A. et al.: Salinity resistance of Citrus seedlings in relation to hydraulic conductance, plasma membrane ATPase and anatomy of the roots. - J. Plant Physiol. 156: 724-730, 2000.

García-Sánchez F., Jifon J.L., Carrajal M. et al.: Gas exchange, chlorophyll and nutrient content in relation to $\mathrm{Na}$ and $\mathrm{Cl}$ accumulation in sunburst mandarin grafted on different rootstock. - Plant Sci. 162: 705-712, 2002.

Genty B., Briantais J.M., Baker N.B.: The relationship between the quantum yield of photosynthetic electrontransport and quenching of chlorophyll fluorescence. - Biochim. Biophys. Acta 990: 87-92, 1989.

Giannopolitis C.N., Ries S.K.: Superoxide dismutases: I. Occurrence in higher plants. - Plant Physiol. 59: 309-314, 1977.

González L., González-Vilar M.: Determination of relative water content. - In: Reigosa M.J. (ed): Handbook of Plant Ecophysiology Techniques. Pp. 207-212. Kluwer Academic, Dordrecht 2001.

Grattan S.R., Grieve C.M.: Mineral element acquisition and growth response of plants grown in saline environment. - Agr. Ecosyst. Environ. 38: 275-300, 1992.

Hamdani S., Gauthier A., Msilini N. et al.: Positive charges of polyamines protect PSII in isolated thylakoid membranes during photoinhibitory conditions. - Plant Cell Physiol. 52: 866-873, 2011.

Hare P.D., Cress W.A.: Metabolic implications of stress-induced proline accumulation in plants. - Plant Growth Regul. 21: 79$102,1997$.

Hedge J.E., Hofreiter B.T.: Estimation of starch by anthrone reagent. - In: Whistler R.L., Be-Miller J.N. (ed.): Methods in Carbohydrate Chemistry. Pp. 9-10. Academic Press, New York 1962.

Hernández J.A., Jiménez A., Mullineaux P., Sevilia F.: Tolerance of pea (Pisum sativum L.) to long term salt stress is associated with induction of antioxidant defences. - Plant Cell Environ. 23: 151-167, 2000.

Hernández J.A., Campillo A., Jiménez A. et al.: Response of antioxidant systems and leaf water relations to $\mathrm{NaCl}$ stress in pea plants. - New Phytol. 141: 241-251, 1999.

Jiménez-Bremont J.F., Becerra-Flora A., Hernández-Lucero E. et al.: Proline accumulation in two bean cultivars under salt stress and the effect of polyamines and ornithine. - Biol. Plantarum 50:763-766, 2006.

Katerji N., van Hoorn J.W., Hamdy A. et al.: Osmotic adjustment of sugarbeets in response to soil salinity and its influence on stomatal conductance, growth and yield. - Agr. Water Manage. 34: 57-69, 1997.

Koshbakht D., Asgharei M.R.: Influence of foliar-applied salicylic acid on growth, gas-exchange characteristics, and chlorophyll fluorescence in citrus under saline conditions. Photosynthetica 53: 410-418, 2015.

Kusano T., Berberich T., Tateda C. et al.: Polyamines: essential factors for growth and survival. - Planta 228: 367-381, 2008.

Larsen M.H., Davis T.D., Evans R.P.: Modulation of protein expression in uniconazole treated soybean in relation to heat stress. - Proc. Plant Growth Regul. Soc. Am. 15: 177-182, 1988.

Leshem Y., Seri L., Levine A.: Induction of phospatidylinositol 3-kinase mediated endocytosis by salt stress leads to intracellular production of reactive oxygen species and salt tolerance. - Plant J. 51: 185-197, 2007.

Lichtenthaler R.K.: Chlorophylls and carotenoids - pigments of photosynthetic biomembranes. - In: Colowick S.P., Kaplan N.O. (ed.): Methods in Enzymology. Vol. 148. Pp. 350-382. Academic Press, San Diego 1987.

Lopatin A.N., Makhina E.N., Nichols C.G.: Potassium channel block by cytoplasmic polyamines as the mechanism of intrinsic rectification. - Nature 372: 366-369, 1994.

Lu C.M., Qiu N.W., Wang B.S. et al.: Salinity treatment shows no effects on photosystem II photochemistry, but increases the resistance of photosystem II to heat stress in halophyte Suaeda salsa. - J. Exp. Bot. 54: 851-860, 2003.

Lutts S., Kinet J.M., Bouharmont J.: Changes in plant response to $\mathrm{NaCl}$ during development of rice (Oryza sativa $\mathrm{L}$.) varieties differing in salinity resistance. - J. Exp. Bot. 46: 1843-1852, 1995.

Lütz C., Navakoudis E., Seidlitz H.K. et al:: Simulated solar irradiation with enhanced UV-B adjust plastid- and thylakoidassociated polyamine changes for UV-B protection. - BBABioenergetics 1710: 24-33, 2005.

Mansfield, T.A., Hetherington, A.M., Atkinson, C.J.: Some current aspects of stomatal physiology. - Annu. Rev. Plant Phys. 41: 55-75, 1990.

Mapelli S., Brambilla I., Radyukina N. et al.: Free and bound polyamines changes in different plants as a consequence of UV-B light irradiation. - Gen. Appl. Plant Physiol. 34: 55-66, 2008.

Marschner H., Cakmak H.: High light intensity enhances chlorosis and necrosis in leaves of zinc, potassium, and magnesium deficient bean (Phaseolus vulgaris) plants. - J. Plant Physio. 134: 308-315, 1989.

Marschner H.: Mineral Nutrition of Higher Plants. Pp. 10-889. Academic Press, San Diego 1995.

Maxwell K., Johnson G.N.: Chlorophyll fluorescence - a practical guide. - J. Exp. Bot. 51: 659-668, 2000.

McCord J.M.: The evolution of free radicals and oxidative stress. - Amer. J. Med. 108: 652-659, 2000.

Misra N., Gupta A.K.: Effect of salt stress on proline metabolism in two high yielding genotypes of green gram. - Plant Sci. 169: 331-339, 2005.

Moya J.L., Primo-Millo E., Talon M.: Morphological factors determining salt tolerance in citrus seedlings: the shoot to root ratio modulates passive root uptake of chloride ions and their accumulation in leaves. - Plant Cell Environ. 22: 1425-1433, 1999.

Munns R., Tester M.: Mechanism of salinity tolerance. - Annu. Rev. Plant Biol. 59: 651-681, 2008.

Nishihara E., Kondo K., Parvez M.M. et al:: Role of 5aminolevulinic acid (ALA) on active oxygen-scavenging system in NaCl-treated spinach (Spinacia oleracea). - Plant Physiol. 160: 1085-1091, 2003.

Noreen Z., Ashraf M., Akram N.A.: Salt-induced regulation of some key antioxidant enzymes and physio-biochemical phenomena in five diverse cultivars of turnip (Brassica rapa L.). - J. Agron. Crop Sci. 196: 273-285, 2010.

Parida A.K., Das A.B.: Salt tolerance and salinity effects on plants: a review. - Ecotoxicol. Environ. Safe. 60: 324-349, 2005.

Parvin S., Lee O.R., Sathiyaraj G. et al.: Spermidine alleviates 
the growth of saline-stressed ginseng seedlings through antioxidative defense system. - Gene 537: 70-78, 2014.

Peltzer D., Dreyer E., Polle A.: Differential temperature dependencies of antioxidative enzymes in two contrasting species. - Plant Physiol. Bioch. 40: 141-150, 2002.

Rao G.G., Rao G.R.: Pigment composition and chlorophyllase activity in pigeon pea (Cajanus indicus Spreng) and Gingelley (Sesamum indicum L.) under $\mathrm{NaCl}$ salinity. - Indian J. Exp. Biol. 19: 768-770, 1981.

Ravindran K.C., Venkatesan K., Balakrishan V. et al.: Restoration of saline land by halophytes for Indian soils. - Soil Biol. Biochem. 39: 2661-2664, 2007.

Rengel Z.: The role of calcium in salt toxicity. - Plant Cell Environ. 15: 625-632, 1992.

Romero-Aranda R., Soria T., Cuartero J.: Tomato plant-water uptake and plant-water relationships under saline growth conditions. - Plant Sci. 160: 265-272, 2001.

Rosales M.A., Rios J.J., Castellano R. et al.: Proline metabolism in cherry tomato exocarp in relation to temperatura and solar radiation. - J. Hortic. Sci. Biotech. 82: 739-744, 2007.

Roy P., Niyogi K., SenGupta D.N. et al.: Spermidine treatment to rice seedlings recovers salinity stress induced damage of plasma membrane and PM-bound $\mathrm{H}^{+}$ATPase in salt-tolerant and salt-sensitive rice cultivars. - Plant Sci. 168: 583-591, 2005.

Ruiz D., Martínez V., Cerdá A.: Citrus response to salinity: growth and nutrient uptake. - Tree Physiol. 17: 141-150, 1997.

Santa-Cruz A., Acosta M., Pérez-Alfocea F. et al.: Changes in free polyamine levels induced by salt stress in leaves of cultivated and wild tomato species. - Physiol. Plantarum 101: 341-346, 1997.

Šetlík S.I., Allakhveridiev L., Nedbal E. et al.: Three type of photosystem II photoinactivation. I. Damaging process on the acceptor side. - Photosynth. Res. 23: 39-48, 1990.

Sfakianaki M., Sfichi L., Kotzabasis K.: The involvement of LHCII-associated polyamines in the response of the photosynthetic apparatus to low temperature. - J. Photoch. Photobio. B. 84: 181-188, 2006.

Shalhevet J.: Plants under salt and water stress. - In: Fowden L., Mansfield T., Stoddart J (ed): Plant Adaptation to Environmental Stress. Pp. 133-154. Chapman and Hall, London-Glasgow-New York-Tokyo-Melbourne-Madras 1993.

Sharma D., Dubey A., Srivastav M. et al.: Effect of putrescine and paclobutrazol on growth, physiochemical parameters., and nutrient acquisition of salt-sensitive citrus rootstock Karna khatta (Citrus karna Raf.) under $\mathrm{NaCl}$ stress. - J. Plant Growth Regul. 30: 301-311, 2011.

Sharma P., Dubey R.S: Protein synthesis by plants under stressful conditions. - In: Pessarakli M. (ed.): Handbook of Plant and Crop Stress. Pp. 465-518. CRC Press, Boca Raton 2010.

Singh A.K, Dubey R.S.: Changes in chlorophyll $a$ and $b$ contents and activities of photosystems I and II in rice seedlings induced by $\mathrm{NaCl}$ - Photosynthetica 31: 489-499, 1995.

Smith T.A.: Polyamines. - Annu. Rev. Plant Physio. 36: 117-143, 1985.

Stępień P., Kłbus G.: Water relations and photosynthesis in Cucumis sativus L. leaves under salt stress. - Biol. Plantarum 50: 610-616, 2006.
Stevens J., Senaratna T., Sivasithamparam K.: Salicylic acid induces salinity tolerance in tomato (Lycopersicon esculentum cv. 'Roma'): associated changes in gas exchange, water relations and membrane stabilisation. - Plant Growth Regul. 49: 77-83, 2006.

Sudhir P., Murthy S.D.S.: Effects of salt stress on basic processes of photosynthesis. - Photosynthetica 42: 481-486, 2004.

Syeed S., Anjum N.A., Nazar R. et al.: Salicylic acid- mediated changes in photosynthesis, nutrients content and antioxidant metabolism in two mustard (Brassica junea L.) cultivars differing in salt tolerance. - Acta Physiol. Plant. 33: 877-886, 2011.

Takahama U., Oniki T.: A peroxidase/phenolics/ascorbate system can scavenge hydrogen peroxide in plant cells. Physiol. Plantarum 101: 845-852, 1997.

Tang W., Newton R.J.: Polyamines reduce salt-inducedoxidative damage by increasing the activities of antioxidant enzymes and decreasing lipid peroxidation in Virginia pine. Plant Growth Regul. 46: 31-43, 2005.

Todorova D., Sergiev I., Alexieva V. et al.: Polyamine content in Arabidopsis thaliana (L.) Heynh during recovery after low and high temperature treatments. - Plant Growth Regul. 51: 185191, 2007.

Tonon G., Kevers C., Faivre-Rampant O. et al.: Effect of $\mathrm{NaCl}$ and mannitol iso-osmotic stresses on proline and free polyamine levels in embryogenic Fraxinus angustifolia callus. - J. Plant Physiol. 161: 701-708, 2004.

Tozlu I., Moore G.A., Guy C.L.: Effect of increasing $\mathrm{NaCl}$ concentration on stem elongation, dry mass production, and macro- and micro- nutrient accumulation in Poncirus trifoliate. - Aust. J. Plant Physiol. 27: 35-42, 2000.

Upchurch R.G.: Fatty acid unsaturation, mobilization and regulation in response of stress to plants. - Biotechnol. Lett. 30: 967-977, 2008.

van Kooten O., Snel J.F.H.: The use of chlorophyll fluorescence nomenclature in plant stress physiology. - Photosynth. Res. 25: 147-150, 1990.

Verma S., Mishra S.N.: Putrescine alleviation of growth in salt stressed Brassica juncea by inducing antioxidative defense system. - J. Plant Physiol. 162: 669-677, 2005.

Wang X., Shi G.X., Xu Q.S. et al.: Exogenous polyamines enhance copper tolerance of Nymphoides peltatum. - J. Plant Physiol. 164: 1062-1070, 2007.

Wi S.J., Kim W.T., Park K.Y.: Overexpression of carnation Sadenosylmethionine decarboxylase gene generates a broad spectrum tolerance to abiotic stresses in transgenic tobacco plants. - Plant Cell. Rep. 25: 1111-1121, 2006.

Yamaguchi K., Takahashi Y., Berberich T. et al.: A protective role for the polyamine spermine against drought stress in Arabidopsis. - Biochem. Biophys. Res. Co. 352: 486-490, 2007.

Zhang L., Zhang Z., Gao H. et al.: Mitochondrial alternative oxidase pathway protects plants against photoinhibition by alleviating inhibition of the repair of photodamaged PSII through preventing formation of reactive oxygen species in Rumex K-1 leaves. - Physiol. Plantarum 143: 396-407, 2011.

Zhu J.K.: Plant salt tolerance. - Trends Plant Sci. 6: 66-71, 2001. Zhu J.K.: Salt and drought stress signal transduction in plants. Annu. Rev. Plant Biol. 53: 247-273, 2002. 